\title{
47TH SASKATCHEWAN CHRISTMAS MAMMAL COUNT - 2019
}

Alan R. Smith

Box 154

Avonlea, SK SOH OCO

alanrandi@sasktel.net

There were only 72 Christmas Mammal Counts to report from this past winter, which is down from the 83 reported from each of the two previous winters. As a result, the 3,191 mammals seen or heard was down from the 3,434 recorded the last winter. As is usual, Whitetailed Deer (1,012 individuals) and Mule Deer (894 animals) were by far the most commonly encountered mammals.

During harsher winters much of the province's Pronghorn population moves south of the border. This past winter, however, Pronghorn were the third most common mammal with 392 animals on seven counts.

Eastern Fox Squirrels continue their expansion along the South Saskatchewan River reaching Outlook; next stop Saskatoon?

Unusual sightings include those of the 13-lined Ground-Squirrel and Fisher. Normally in hibernation, the ground-squirrel was seen in the Pipestone Creek valley during the count period of the Broadview Count. Usually a boreal forest mammal, the Fisher was seen at Round Lake in the Qu'Appelle Valley.

With 13 species seen or heard, Odessa and Indian Head tied for the most species on a count. No new species were added this past winter so the all-time provincial total remains at 51 species seen or heard (plus three species found dead and two others recorded only on the basis of tracks).

Although certainly neither a bird or mammal, two Leopard Frogs in a spring on the Cypress Hills Provincial Park count are noteworthy. Incredibly they have been reported on several previous counts in the Park.

For information on participants, weather, coverage and location of Christmas Mammal Counts, see the Christmas Bird Count summary in this issue.

\section{Explanation of entries in Table 1.}

The number of mammals actually seen or heard on count day is treated separately from those recorded by other means, or those recorded during count period (14 December to 5 January) but not on count day. Numbers of individuals seen or heard are given in Table 1 and are tallied in the first line of totals at the bottom of the table. The number of species they represent is given in the second line.

For species only detected by tracks or by other means, or that are seen or heard only in the count period but not on count day, no numbers of individuals is given in Table 1. Species detected only by tracks are indicated by ' $\mathrm{t}$ ' in the table; those detected only by other means: dead animals ' $m$ ', more clearly identifiable chewing or digging ' $d$ ', dens or lodges 'L' (including Muskrat push-ups) and by smell 's.' Species detected by any means during the count period, but not on count day are indicated by ' $c$ ' in the table. These additional species are tallied in lines 3, 4 and 5 at the bottom of the table. If a mammal is reported as member of a species group (i.e. mouse species, deer species), it is counted as a species only if no other species in this group has been definitely recorded. The columns at the end of the table give totals for each species.

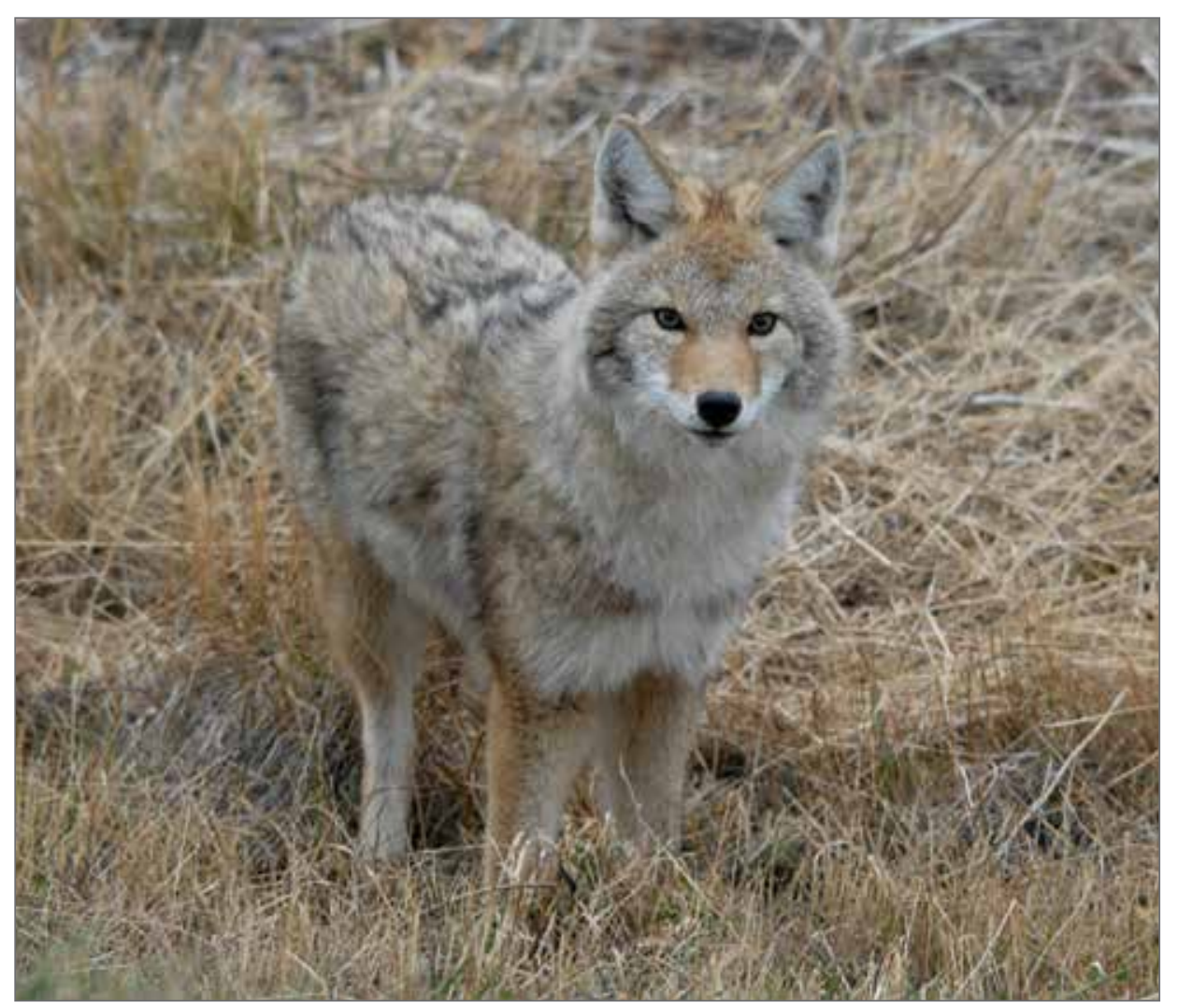

Coyote. Photo credit: Randy McCulloch. 
TABLE 1. 47th Saskatchewan Christmas Mammal Count - 2019.

\begin{tabular}{|c|c|c|c|c|c|c|c|c|c|c|c|c|c|c|c|c|c|c|c|c|}
\hline SPECIES & 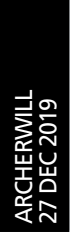 & 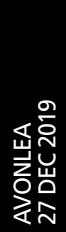 & 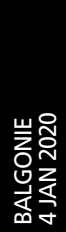 & 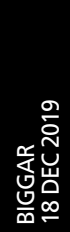 & 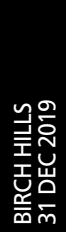 & 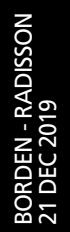 & 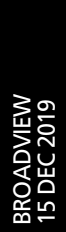 & 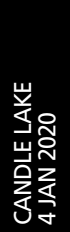 & 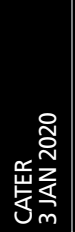 & 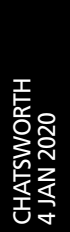 & 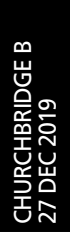 & 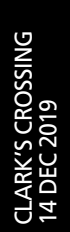 & 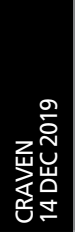 & 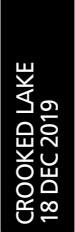 & 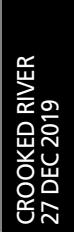 & 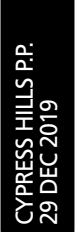 & 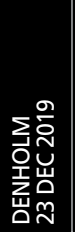 & 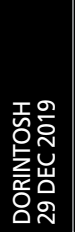 & 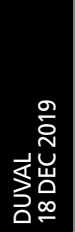 & 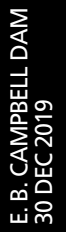 \\
\hline \multicolumn{21}{|l|}{ Short-tailed Shrew } \\
\hline Shrew species & & & & & & & & & & & & 1 & & & & & & & & \\
\hline \multicolumn{21}{|l|}{ Eastern Cottontail } \\
\hline Nuttall's Cottontail & & & & & & & & & & & & & & & $\mathrm{t}$ & $\mathrm{t}$ & & & $\mathrm{t}$ & \\
\hline Snowshoe Hare & 1 & & $\mathrm{t}$ & & & $\mathrm{t}$ & $\mathrm{t}$ & $\mathrm{t}$ & $\mathrm{t}$ & & & $\mathrm{t}$ & & & & 1 & $\mathrm{t}$ & $\mathrm{t}$ & $\mathrm{t}$ & $\mathrm{t}$ \\
\hline White-tailed Jack Rabbit & & & 1 & & & 1 & $\mathrm{t}$ & & & & & 1 & $\mathrm{t}$ & & & & c & & & \\
\hline 13-lined Ground Squirrel & & & & & & & c & & & & & & & & & & & & & \\
\hline \multicolumn{21}{|l|}{ Eastern Grey Squirrel } \\
\hline Eastern Fox Squirrel & & 4 & 22 & & & & 1 & & & & & & 7 & & & & & & 1 & \\
\hline American Red Squirrel & 2 & & & & $\mathrm{t}$ & 1 & 1 & 13 & & 2 & 3 & 2 & 3 & 2 & & 13 & & $\mathrm{t}$ & & 16 \\
\hline Northern Flying-Squirrel & & & & & & & & & & & & & & & & & 1 & & & \\
\hline \multicolumn{21}{|l|}{ Northern Pocket Gopher } \\
\hline American Beaver & & & & & & & $\mathrm{L}$ & $\mathrm{L}$ & $\mathrm{L}$ & $\mathrm{L}$ & & & & & & & & $\mathrm{L}$ & $\mathrm{L}$ & \\
\hline Deer Mouse & & & & & $\mathrm{t}$ & & $\mathrm{t}$ & & & & & & & & & & c & & & \\
\hline Muskrat & & & & $\mathrm{L}$ & & & & & $\mathrm{L}$ & & & & & 1 & & & & $\mathrm{~L}$ & $\mathrm{~L}$ & \\
\hline $\begin{array}{l}\text { Gapper's Red-Backed } \\
\text { Vole }\end{array}$ & & & & & & & & & & & & & & & & & 1 & & & \\
\hline Meadow Vole & & & & & & & & & & & & & & & & & & & & 1 \\
\hline Vole species & & & & $\mathrm{t}$ & $\mathrm{t}$ & $\mathrm{t}$ & & & & & & $\mathrm{t}$ & $\mathrm{t}$ & & 1 & & & & $\mathrm{t}$ & $\mathrm{t}$ \\
\hline House Mouse & & & & & & & & & & 1 & & & & & & & & & & \\
\hline Mouse species & $\mathrm{t}$ & & $\mathrm{t}$ & & $\mathrm{t}$ & 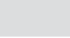 & & & & & & & $\mathrm{t}$ & & 1 & $\mathrm{t}$ & & & $\mathrm{t}$ & $\mathrm{t}$ \\
\hline American Porcupine & & & & 2 & & $\mathrm{t}$ & & & & & & 1 & & . & & . & & $\mathrm{t}$ & 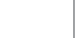 & \\
\hline Coyote & C & & & 3 & $\mathrm{t}$ & 9 & 1 & & 1 & & & 8 & 6 & $\mathrm{t}$ & & 1 & 2 & $\mathrm{t}$ & 1 & \\
\hline Gray Wolf & & & & & & & & $\mathrm{t}$ & & & & & & & & & & $\mathrm{t}$ & . & $\mathrm{t}$ \\
\hline Red Fox & & & 1 & $\mathrm{t}$ & $\mathrm{t}$ & & $\mathrm{t}$ & $\mathrm{t}$ & $\mathrm{t}$ & & & & $\mathrm{t}$ & $\mathrm{t}$ & & 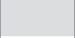 & $\mathrm{t}$ & c & $\mathrm{t}$ & $\mathrm{t}$ \\
\hline Raccoon & 2 & & & & & & & & & & & & & & & & & & & 1 \\
\hline \multicolumn{21}{|l|}{ Fisher } \\
\hline Ermine & & & & & 1 & & & & & & & & & & & & & & & \\
\hline Long-tailed Weasel & & & & & & & & & & & & & & & & & & & $\mathrm{t}$ & \\
\hline \multicolumn{21}{|l|}{ Least Weasel } \\
\hline Weasel species & c & & & $\mathrm{t}$ & & $\mathrm{t}$ & & $\mathrm{t}$ & & & & 1 & & 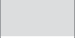 & & & & $\mathrm{t}$ & & $\mathrm{t}$ \\
\hline American Mink & & & & & & & $\mathrm{t}$ & & & 1 & & & & 1 & & & . & $\mathrm{t}$ & & \\
\hline American Badger & & & & $d$ & & & & & & & & & & & & & $d$ & & & \\
\hline Striped Skunk & 1 & & & & & & & & & & & & & & & & & & & \\
\hline River Otter & & & & & & & c & 1 & & & & & & & & & & $\mathrm{t}$ & & $\mathrm{t}$ \\
\hline \multicolumn{21}{|l|}{ Canada Lynx } \\
\hline Mule Deer & & 8 & 26 & 16 & & 11 & c & & & & & 7 & 25 & & & & 7 & & 11 & \\
\hline White-tailed Deer & 14 & 13 & 5 & 16 & $\mathrm{t}$ & 15 & 6 & 25 & 1 & 8 & 5 & 29 & 57 & $\mathrm{t}$ & 2 & 39 & 23 & 48 & 8 & 10 \\
\hline Moose & $\mathrm{t}$ & & $\mathrm{t}$ & & & & 1 & & & & 3 & & & $\mathrm{t}$ & & & c & $\mathrm{t}$ & & \\
\hline Elk & & & & & & & & & & & & & & & & c & & & & \\
\hline Pronghorn & & & & & & & & & & & & & 1 & & & & & & & \\
\hline $\begin{array}{l}\text { TOTALS SEEN/HEARD ON } \\
\text { COUNT DAY }\end{array}$ & 20 & 25 & 55 & 37 & 1 & 37 & 10 & 39 & 2 & 12 & 11 & 50 & 99 & 4 & 3 & 54 & 34 & 48 & 21 & 28 \\
\hline TOTAL SPECIES SEEN/HEARD & 5 & 3 & 5 & 4 & 1 & 5 & 5 & 3 & 2 & 4 & 3 & 8 & 6 & 3 & 3 & 4 & 5 & 1 & 4 & 4 \\
\hline $\begin{array}{l}\text { TOTAL SPECIES RECORDED } \\
\text { BY TRACKS }\end{array}$ & 2 & 0 & 3 & 3 & 6 & 4 & 5 & 4 & 2 & 0 & 0 & 2 & 3 & 4 & 1 & 3 & 2 & 9 & 6 & 5 \\
\hline $\begin{array}{l}\text { TOTAL SPECIES OTHERWISE } \\
\text { RECORDED }\end{array}$ & 0 & 0 & 0 & 2 & 0 & 0 & 1 & 1 & 2 & 1 & 0 & 0 & 0 & 0 & 0 & 0 & 2 & 2 & 2 & 0 \\
\hline $\begin{array}{l}\text { SPECIES RECORDED COUNT } \\
\text { PERIOD }\end{array}$ & 2 & 0 & 0 & 0 & 0 & 0 & 3 & 0 & 0 & 0 & 0 & 0 & 0 & 0 & 0 & 1 & 3 & 1 & 0 & 0 \\
\hline $\begin{array}{l}\text { TOTAL SPECIES COUNT } \\
\text { PERIOD AND DAY }\end{array}$ & 9 & 3 & 8 & 9 & 7 & 9 & 14 & 8 & 6 & 5 & 3 & 10 & 9 & 7 & 4 & 8 & 12 & 13 & 12 & 9 \\
\hline
\end{tabular}




\section{SPECIES}

Short-tailed Shrew

Shrew species

Eastern Cottontail

Nuttall's Cottontail

Snowshoe Hare

White-tailed Jack Rabbit

13-lined Ground Squirrel

Eastern Grey Squirrel

Eastern Fox Squirrel

American Red Squirrel

Northern Flying-Squirrel

Northern Pocket Gopher

American Beaver

Deer Mouse

Muskrat

Gapper's Red-Backed

Vole

Meadow Vole

Vole species

House Mouse

Mouse species

American Porcupine

Coyote

Gray Wolf

Red Fox

Raccoon

Fisher

Ermine

Long-tailed Weasel

Least Weasel

Weasel species

American Mink

American Badger

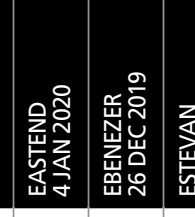

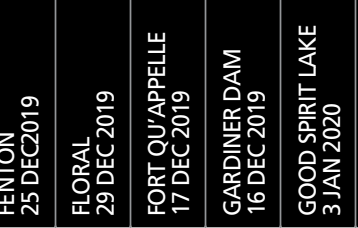

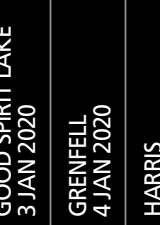

触

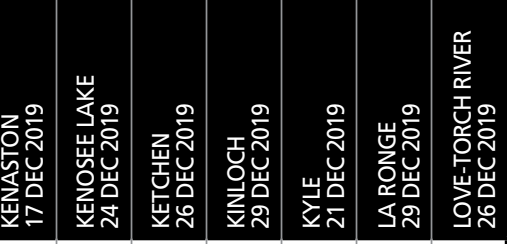

\section{Striped Skunk}

River Otter

Canada Lynx

Mule Deer

White-tailed Deer

\begin{tabular}{|l|l|}
\hline & 4 \\
\hline
\end{tabular}

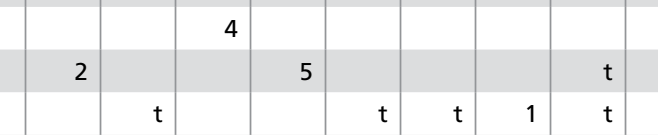

\begin{tabular}{|c|c|c|}
\hline & & \\
\hline
\end{tabular}

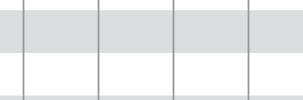


SPECIES

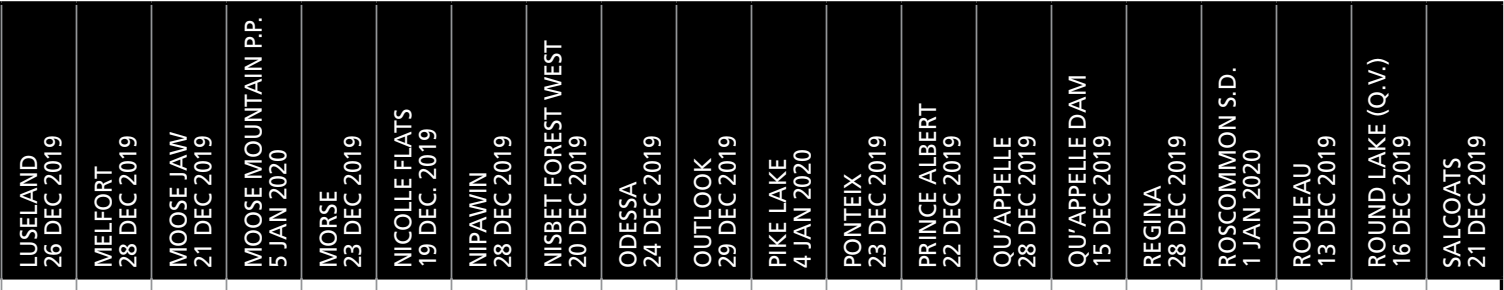

Short-tailed Shrew

Shrew species

Eastern Cottontail

Nuttall's Cottontail

Snowshoe Hare

White-tailed Jack Rabbit

13-lined Ground Squirrel

Eastern Grey Squirrel

Eastern Fox Squirrel

American Red Squirrel

Northern Flying-Squirrel

Northern Pocket Gopher

American Beaver

Deer Mouse

Muskrat

Gapper's Red-Backed

Vole

Meadow Vole

Vole species

House Mouse

Mouse species

American Porcupine

Coyote

Gray Wolf

Red Fox

Raccoon

Fisher

Ermine

Long-tailed Weasel

Least Weasel

Weasel species

American Mink

American Badger

Striped Skunk

River Otter

Canada Lynx

Mule Deer

White-tailed Deer

Moose

Elk

Pronghorn

\begin{tabular}{|l|r|r|r|r|r|r|}
\hline & 19 & & & 10 & & \\
\hline
\end{tabular}

\begin{tabular}{|l|l|l|l|r|}
\hline 2 & & & & \\
\hline
\end{tabular}

\begin{tabular}{|l|l|l|l|}
\hline & & & \\
\hline
\end{tabular}

\begin{tabular}{|l|l|l|l|l|l|}
\hline & & & \\
\hline
\end{tabular}

\begin{tabular}{|c|c|c|c|c|c|c|c|c|c|c|c|c|c|c|c|c|c|c|c|c|}
\hline $\begin{array}{l}\text { TOTALS SEEN/HEARD } \\
\text { ON COUNT DAY }\end{array}$ & 7 & 22 & 58 & 2 & 189 & 121 & 25 & 6 & 70 & 2 & 40 & 81 & 23 & 30 & 128 & 151 & 18 & 2 & 13 & 13 \\
\hline TOTAL SPECIES SEEN/HEARD & 4 & 2 & 4 & 1 & 7 & 6 & 3 & 2 & 13 & 2 & 5 & 3 & 6 & 5 & 7 & 6 & 7 & 2 & 5 & 3 \\
\hline $\begin{array}{l}\text { TOTAL SPECIES } \\
\text { RECORDED BY TRACKS }\end{array}$ & 0 & 0 & 0 & 0 & 0 & 5 & 7 & 1 & 0 & 0 & 4 & 1 & 9 & 2 & 2 & 5 & 6 & 0 & 1 & 0 \\
\hline $\begin{array}{l}\text { TOTAL SPECIES } \\
\text { OTHERWISE RECORDED }\end{array}$ & 0 & 0 & 0 & 0 & 1 & 1 & 0 & 0 & 0 & 0 & 0 & 1 & 3 & 2 & 0 & 1 & 1 & 1 & 1 & 0 \\
\hline $\begin{array}{l}\text { SPECIES RECORDED } \\
\text { COUNT PERIOD }\end{array}$ & 0 & 0 & 2 & 0 & 0 & 0 & 0 & 0 & 0 & 1 & 1 & 0 & 0 & 9 & 0 & 1 & 0 & 0 & 0 & 0 \\
\hline $\begin{array}{l}\text { TOTAL SPECIES } \\
\text { COUNT PERIOD AND DAY }\end{array}$ & 4 & 2 & 6 & 1 & 8 & 12 & 10 & 3 & 13 & 3 & 10 & 5 & 18 & 18 & 9 & 13 & 14 & 3 & 7 & 3 \\
\hline
\end{tabular}


SPECIES

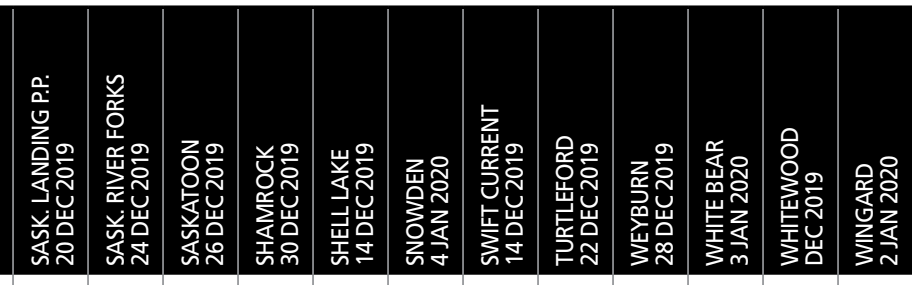

Short-tailed Shrew

Shrew species

Eastern Cottontail

Nuttall's Cottontail

Snowshoe Hare

White-tailed Jack Rabbit

13-lined Ground Squirrel

Eastern Grey Squirrel

Eastern Fox Squirrel

American Red Squirrel

Northern Flying-Squirrel

Northern Pocket Gopher

American Beaver

Deer Mouse

Muskrat

Gapper's Red-Backed

Vole

Meadow Vole

Vole species

House Mouse

Mouse species

American Porcupine

Coyote

Gray Wolf

Red Fox

Raccoon

Fisher

Ermine

Long-tailed Weasel

Least Weasel

Weasel species

American Mink

American Badger

Striped Skunk

River Otter

\section{Canada Lynx}

Mule Deer

White-tailed Deer

Moose

Elk

Pronghorn

TOTALS SEEN/HEARD

ON COUNT DAY

TOTAL SPECIES SEEN/HEARD

TOTAL SPECIES

RECORDED BY TRACKS

TOTAL SPECIES

OTHERWISE RECORDED

SPECIES RECORDED

COUNT PERIOD

TOTAL SPECIES
COUNT PERIOD AND DAY

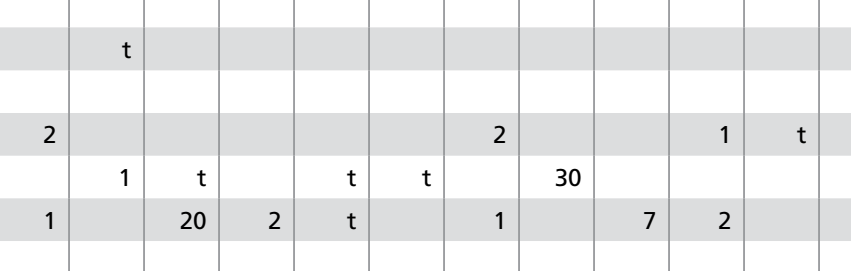

\begin{tabular}{|l|l|l|l|l|l|l|}
\hline & & & & & & 21 \\
\hline
\end{tabular}

21
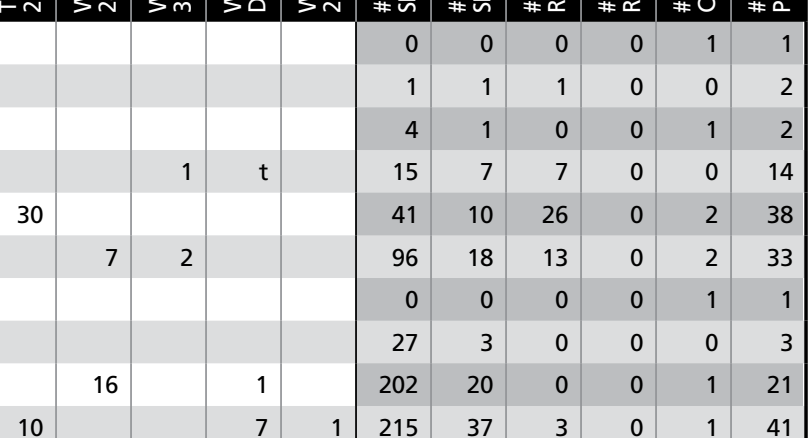

\begin{tabular}{|r|r|r|r|r|r|}
\hline 4 & 1 & 0 & 0 & 1 & 2 \\
\hline 15 & 7 & 7 & 0 & 0 & 14 \\
\hline 41 & 10 & 26 & 0 & 2 & 38 \\
\hline
\end{tabular}

\begin{tabular}{r|r|r|r|r|r|}
\hline 96 & 18 & 13 & 0 & 2 & 33 \\
\hline
\end{tabular}

\begin{tabular}{|r|r|r|r|r|r|}
\hline 0 & 0 & 0 & 0 & 1 & 1 \\
\hline
\end{tabular}

\begin{tabular}{|l|l|l|l|l|l|}
\hline 27 & 3 & 0 & 0 & 0 & 3 \\
\hline
\end{tabular}

\begin{tabular}{|l|l|l|l|l|r|}
202 & 20 & 0 & 0 & 1 & 21 \\
\hline
\end{tabular}

\begin{tabular}{|r|r|r|r|r|r|}
\hline 215 & 37 & 3 & 0 & 1 & 41 \\
\hline
\end{tabular}

\begin{tabular}{|l|l|l|l|l|l|}
2 & 2 & 0 & 0 & 0 & 2 \\
\hline
\end{tabular} d

\begin{tabular}{|l|l|l|l|l|l|}
\hline 0 & 0 & 0 & 3 & 1 & 4 \\
\hline
\end{tabular}

L

\begin{tabular}{|l|l|r|}
\hline & 1 & $\mathrm{t}$ \\
\hline $\mathrm{L}$ & & $\mathrm{L}$
\end{tabular}

\begin{tabular}{|r|r|r|r|r|r|r|}
\hline 0 & 0 & 0 & 19 & 2 & 21 \\
\hline 1 & 1 & 7 & 0 & 2 & 10 \\
\hline
\end{tabular}

\begin{tabular}{|r|r|r|r|r|r|}
\hline 1 & 1 & 7 & 0 & 2 & 10 \\
\hline 4 & 2 & 0 & 10 & 0 & 12 \\
\hline
\end{tabular}

\begin{tabular}{l|l|l|l} 
& \\
$\mathrm{t}$ & $\mathrm{t}$
\end{tabular}

\begin{tabular}{|l|l|l|l|l|l|}
1 & 1 & 1 & 0 & 1 & 3 \\
\hline
\end{tabular}

\begin{tabular}{|r|r|r|r|r|r|}
\hline 1 & 1 & 4 & 0 & 0 & 5 \\
\hline 5 & 4 & 21 & 0 & 0 & 25 \\
\hline
\end{tabular}

\begin{tabular}{|l|l|l|l|l|l|}
\hline 1 & 1 & 0 & 0 & 0 & 1 \\
\hline
\end{tabular}

\begin{tabular}{|r|r|r|r|r|r|}
\hline 1 & 1 & 22 & 0 & 0 & 23 \\
\hline
\end{tabular}

\begin{tabular}{|l|l|l|l|l|l|}
\hline 13 & 9 & 11 & 3 & 0 & 23 \\
\hline
\end{tabular}

\begin{tabular}{|r|r|r|r|r|r|}
\hline 147 & 43 & 12 & 0 & 3 & 58 \\
\hline
\end{tabular}

\begin{tabular}{|r|r|r|r|r|r|}
\hline 0 & 0 & 5 & 0 & 1 & 6 \\
\hline
\end{tabular}

\begin{tabular}{|l|r|r|r|r|r|}
\hline 35 & 15 & 19 & 0 & 2 & 36 \\
\hline
\end{tabular}

\begin{tabular}{|l|l|l|l|l|l|}
9 & 3 & 2 & 2 & 0 & 7 \\
\hline
\end{tabular}

\begin{tabular}{|l|l|l|l|l|l|}
1 & 1 & 1 & 0 & 0 & 2 \\
\hline 4 & 3 & 2 & 0 & 1 & 6 \\
\hline
\end{tabular}

\begin{tabular}{|l|l|l|l|l|l|}
\hline 4 & 3 & 2 & 0 & 1 & 6 \\
\hline
\end{tabular}

\begin{tabular}{|l|l|l|l|l|l|}
\hline 0 & 0 & 5 & 0 & 1 & 6 \\
\hline 0 & 0 & 3 & 0 & 0 & 3 \\
\hline
\end{tabular}

\begin{tabular}{|r|r|r|r|r|r|}
\hline 2 & 2 & 13 & 0 & 2 & 17 \\
\hline
\end{tabular}

\begin{tabular}{|l|l|l|l|l|l|}
3 & 3 & 5 & 0 & 2 & 10 \\
\hline
\end{tabular}

\begin{tabular}{|r|r|r|r|r|r|}
\hline 1 & 1 & 0 & 12 & 0 & 13 \\
\hline 4 & 4 & 2 & 1 & 0 & 7 \\
\hline
\end{tabular}

\begin{tabular}{|l|l|l|l|l|l|}
\hline 6 & 3 & 2 & 1 & 0 & 7 \\
\hline & 3 & 3 & 0 & 1 & 7 \\
\hline
\end{tabular}

\begin{tabular}{|r|r|r|r|r|r|}
\hline 0 & 0 & 1 & 0 & 0 & 1 \\
\hline
\end{tabular}

\begin{tabular}{|l|l|l|l|l|r|}
\hline 894 & 42 & 0 & 0 & 2 & 44 \\
\hline
\end{tabular}

\begin{tabular}{|l|l|l|l|l|l|}
\hline 1012 & 59 & 4 & 0 & 1 & 64 \\
\hline
\end{tabular}

\begin{tabular}{|l|r|r|r|r|r|}
\hline 31 & 14 & 14 & 0 & 3 & 31 \\
\hline
\end{tabular}

\begin{tabular}{|r|r|r|r|r|r|}
\hline 21 & 2 & 4 & 0 & 2 & 8 \\
\hline 392 & 7 & 0 & 0 & 1 & 8 \\
\hline
\end{tabular}

\begin{tabular}{|r|r|r|r|}
\hline 7 & 1 & 2 \\
\hline 20 & & & $\mathrm{t}$ \\
\hline
\end{tabular}

3191

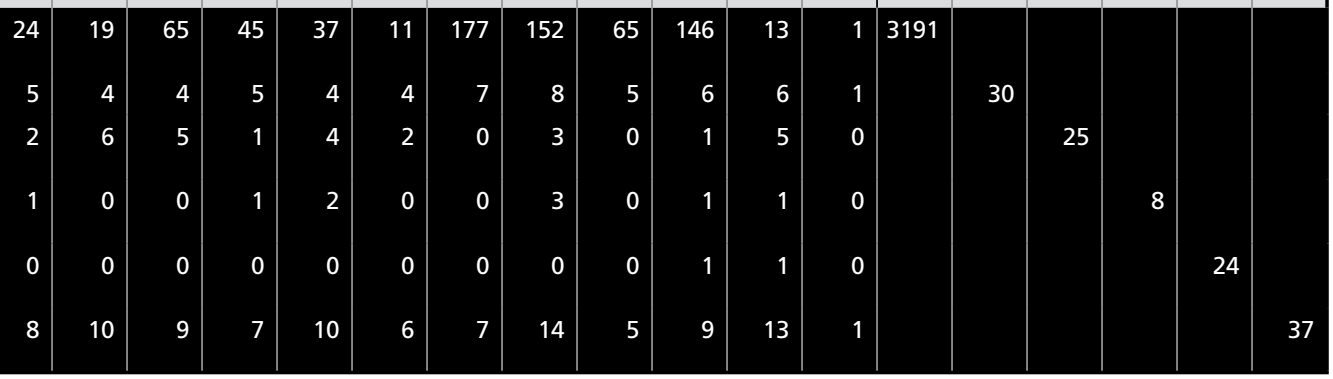

